\title{
Antitumor activity of adenoviral vector containing T42 and $4 \times T 42$ peptide gene through inducing apoptosis of tumor cells and suppressing angiogenesis
}

\author{
XIONG ZHANG $^{1 *}$, DONG-DONG QI $^{2 *}$, TING-TING ZHANG $^{3}$, QING-XIN CHEN ${ }^{4}$, GUANG-ZHI WANG $^{5}$, \\ GUANG-YU SUI ${ }^{6}$, XUE-WEI HAO ${ }^{7}$, SHOULI SUN ${ }^{7}, \mathrm{XUE} \mathrm{SONG}^{7}$ and YING-LI CHEN ${ }^{7}$ \\ ${ }^{1}$ Department of Psychiatry and ${ }^{2}$ Clinical Laboratory, Hulunbeier Mental Health Center, Yakeshi, Inner Mongolia 022150; \\ ${ }^{3}$ Department of Urinary Surgery, General Hospital of Daqing Oil Field, Daqing, Heilongjiang 163000; \\ ${ }^{4}$ Department of Pharmacology, Harbin Medical University, Harbin, Heilongjiang 150000; \\ ${ }^{5}$ Department of Pathology, The Fifth Affiliated Hospital, Harbin Medical University; \\ ${ }^{6}$ Clinical Laboratory, Chinese Medicine Hospital of Daqing; ${ }^{7}$ Department of Immunology, \\ College of Medical Laboratory Science and Technology, Harbin Medical University-Daqing, \\ Daqing, Heilongjiang 163319, P.R. China
}

Received February 13, 2014; Accepted October 20, 2014

DOI: $10.3892 / \mathrm{mmr} .2014 .2910$

\begin{abstract}
The T42 peptide, generated from two active fragments of tumstatin, has been shown to have anti-tumor activity. The adenoviral vector is the most frequently used vector in research and clinical trials for gene therapy. In the present study, the anti-tumor activity of the T42 peptide and quadruple T42 (4xT42) peptide adenoviral vectors were elucidated for the first time, to the best of our knowledge. Human embryonic kidney 293 cells were infected with plasmid adenovirus (pAd)-enhanced green fluorescent protein (EGFP)-T42 or pAd-EGFP-4xT42 and the expression of the T42 and 4xT42 genes was confirmed by the identification of GFP expression and reverse transcription polymerase chain reaction experiments. The anti-cancer effects of pAd-EGFP-T42 and pAd-EGFP-4xT42 on breast cancer cells in vivo and in vitro were subsequently investigated.
\end{abstract}

Correspondence to: Mr. Ying-Li Chen, Department of Immunology, College of Medical Laboratory Science and Technology, Harbin Medical University-Daqing, 39 Xinyang Road, Daqing, Heilongjiang 163319, P.R. China

E-mail: hydwtm@126.com

*Contributed equally

Abbreviations: CPE, cytopathic effect; DMEM, Dulbecco's modified Eagle's medium; DMSO, dimethyl sulfoxide; EGFP, enhanced green fluorescent protein; FBS, fetal bovine serum; MOI, multiplicity of infection; MVD, microvascular density; PBS, phosphate-buffered saline; Pfu, plaque-forming unit; RT-PCR, reverse-transcription polymerase chain reaction

Key words: T42 peptide, 4xT42 peptide, tumstatin, adenovirus vector, green fluorescent protein
The results indicated that the packaging of the recombinant adenoviruses with the viral titer was successful, following purification at $5 \times 10^{9}$ plaque forming units $/ \mathrm{ml}$. The results also revealed that the recombinant adenoviruses promoted apoptosis in MCF-7 breast cancer cells and inhibited cancer growth. Through the analysis of caspase-3, B-cell lymphoma 2 (Bcl-2) and $\mathrm{Bcl}-2$-associated $\mathrm{X}$ protein expression, it was demonstrated that the T42/4xT42 peptide may induce apoptosis via the mitochondrial pathway. In addition, mouse xenograft experiments confirmed that the T42 peptide inhibited tumor growth and reduced angiogenesis in vivo. In conclusion, the results of the present study indicated that the T42 and 4xT42 peptide genes, transfected by a recombinant adenovirus, may provide a potential novel strategy for the treatment of breast cancer.

\section{Introduction}

Breast cancer is a significant health concern amongst females. Gene therapy as a treatment for cancer has received increasing attention following the development of efficient molecular techniques $(1,2)$. Studies have demonstrated that the development of novel blood vessels surrounding tumors provide nutrition and remove metabolic products, which promotes tumor growth and induces tumor metastasis (3). Inhibition of angiogenesis results in the apoptosis or necrosis of tumor cells (4). Angiogenesis inhibitors target vascular endothelial cells, which are genetically stable and invulnerable to mutation (5). In addition, endogenous angiogenesis inhibitors are highly selective and specifically inhibit the proliferation of endothelial cells within lesions (6). Therefore, the use of such inhibitors has become an effective strategy for the treatment of various types of cancer (7).

Tumstatin was discovered as an endogenous angiogenesis inhibitor, exhibiting marked anti-tumor activity in 2003 (8). Tumstatin exerts its effects by inhibiting protein synthesis 
in vascular endothelial cells and inducing apoptosis, thereby inhibiting angiogenesis. In addition, tumstatin inhibits the proliferation of tumor cells and promotes their apoptosis $(9,10)$. Furthermore, tumstatin is able to inhibit tumor growth and the associated pathological angiogenesis without affecting physiological angiogenesis (11).

Studies regarding the safety and effectiveness of active fragments of tumstatin have attracted attention worldwide as tumstatin was demonstrated to be a pathogenic antigen with a high molecular weight, which may result in glomerulonephritis and lung hemorrhage (12). In previous studies by our group, amino acids 185-203 at the $\mathrm{C}$ terminus were successfully transformed into the T21 peptide, which demonstrated anti-angiogenic activity, and amino acids 75-95 at the N-terminus were transformed into the T19 peptide, which exhibited anti-cancer activity. The lower molecular weights of these fragments reduced the immunogenicity and improved the safety of tumstatin. The T21 peptide was subsequently reconstructed with the arginylglycylaspartic acid (RGD)-peptide sequence, which was shown to inhibit the proliferation and migration of tumor cells. The two active fragments of tumstatin, T21RGD and T19 peptide, were ligated via a soft linker to form the recombinant T42 peptide, which was constructed based on previous studies $(13,14)$. The activity of the constructed peptide was hypothesized to be higher than that of any of the single fragments used in previous studies. Previous studies demonstrated that the tumstatin-associated T42 and quadruple (4x)T42 peptide had significant anti-tumor activity $(15,16)$ and inhibited the growth and proliferation of the human cancer cell line HepG2 while promoting apoptosis (9).

An adenovirus is a non-integrated double-stranded DNA virus which is widely distributed throughout nature. Although certain types of adenovirus cause acute gastrointestinal, pulmonary or eye infection in humans (17), the adenovirus used in gene therapy is comparatively safe and carries a low risk of pathogenesis, teratogenesis or carcinogenesis. As the most widely used vector in clinical trials for gene therapy, the adenoviral vector has also been extensively used in research. Adenovirus-mediated gene therapy is conventionally conducted in vivo $(18,19)$.

In previous experiments by our group, purified T42 peptide was applied to MCF-7 cells in vitro. T42 was found to have an anti-tumor effect (data is not shown); however, the metabolic rate of such small molecular proteins in the body was so rapid that high, repeated doses were required. Therefore, it was hypothesized that expression of the 4xT42 peptide gene may also have anti-tumor effects and circumvent the requirement for high, repeated doses. In the present study, it was demonstrated for the first time, to the best of our knowledge, that adenoviral vectors containing the T42 or quadruple T42 (4xT42) genes had antitumor activity. By artificially synthesizing the T42 peptide gene, the adenoviral vectors plasmid adenovirus-enhanced green fluorescent protein-T42 (pAd-EGFP-T42) and pAd-EGFP-4xT42 were successfully constructed using the isocaudarner ligation technique. Subsequently, 293 cells were infected with pAd-EGFP-T42 and pAd-EGFP-4xT42. To confirm the expression of the T42 and 4xT42 genes, GFP expression levels were monitored by fluorescence microscopy and the expression of T42 was assessed by reverse transcription polymerase chain reaction (RT-PCR). The anti-cancer effects of pAd-EGFP-T42/pAd-EGFP-4xT42 on breast cancer cells in vitro as well as in vivo were also preliminarily investigated. In addition, the mechanism underlying the anticancer activity was further investigated.

\section{Materials and methods}

Cells, drugs and animals. The human embryonic kidney 293 cell line and the human breast cancer cell line MCF-7 were obtained from the American Type Culture Collection (Manassas, VA, USA). The cells were maintained in Dulbecco's modified Eagle's medium (DMEM; Invitrogen Life Technologies, Carlsbad, CA, USA) supplemented with $10 \%$ fetal bovine serum (FBS; Sijiqing Biological Engineering Materials Co., Hangzhou, China) at $37^{\circ} \mathrm{C}$ in $5 \% \mathrm{CO}_{2}$. Female $\mathrm{BALB} / \mathrm{c}$ immunodeficient nude mice that were $6-8$ weeks old and weighed 18-20 $\mathrm{g}$ were used. The mice were maintained under controlled conditions of humidity $(50 \pm 10 \%)$, light $\left(12 / 12 \mathrm{~h}\right.$ light/dark cycle) and temperature $\left(23 \pm 2^{\circ} \mathrm{C}\right)$, had ad libitum access to water and were fed a pelleted basal diet. All animal handling and experimental procedures were performed in accordance with the Guide for the Care and Use of Laboratory Animals published by the National Institutes of Health (NIH Published No. 85-23, revised 1996; Bethesda, MA, USA). The present study was approved by the Medical Ethics Committee of Heilongjiang province (Daqing, China),

Construction, collection, amplification and identification of pAd-EGFP-T42, pAd-EGFP-4xT42 and $p A d-E G F P$. The T42 gene was synthesized artificially according to the reference sequence of protein MPFLFCNVNDVCNFASRGDYSGGASPFLECHGRGTCN YYSNS (AXYBIO BIO-TECH CO., Changsha, China). Recognition sites for the restriction enzymes BamHI and $B g l$ II-EcoRI were added to the $5^{\prime}$ and $3^{\prime}$ ends of the sequence, respectively. PCR followed by restriction digestion and subsequent clone screening was used to generate pEC3.1(+)-EGFP-T42 and pEC3.1(+)-EGFP-4xT42 from the recombinant plasmids pEC3.1(+)-T42 and pEC3.1(+)-4xT42. The pAd-EGFP-T42, pAd-EGFP-4xT42 and pAd-EGFP plasmids were constructed by transferring the EGFP-4xT42, EGFP-T42 and EGFP expression cassettes into pAd-BL-Dest adenoviral vectors (Invitrogen Life Technologies) using homologous recombination in vitro by LR reaction (20). The vector was then linearized by single enzyme digestion with restriction endonuclease Pac I (New England Biolabs, Inc., Ipswich, MA, USA) for transfection. The linearized vector was transfected into 293 cells and the GFP expression levels were examined to determine whether the pAd)-EGFP-T42, pAd-EGFP-4xT42 and pAd-EGFP had been successfully recombined. The successfully transfected 293 cells were collected when a marked cytopathogenic effect was observed and $>50 \%$ of the cells were floating. The cells were subjected to three rounds of freeze-thaw cycles and centrifugation at $12,000 \mathrm{x} \mathrm{g}$ for $10 \mathrm{~min}$ at $4^{\circ} \mathrm{C}$, and the viral fluid was collected. The adenovirus was purified using the Adenovirus Purification Maxi kit (ClonTech Laboratories, Inc., Mountain View, CA, USA). RT-PCR was subsequently used to determine the viral 
load (20), using a kit purchased from Takara Bio, Inc. (Otsu, Japan). To measure the viral titer, $20 \mu \mathrm{l}$ viral sample was serially diluted and cultivated for two days in a 24-well plate. The number of cells expressing GFP fluorescence was counted under a fluorescence microscope (Nikon-TE2000-U; Nikon Corporation, Tokyo, Japan). The viral titer was calculated using the following equation: (Number of cells with fluorescence) $\mathrm{x}$ (dilution factor)/(quantity of viruses inoculated).

Plaque assay. The plaque assay utilized a confluent monolayer of 293 cells plated in six-well plates (NEST Science Co., Ltd., Shanghai, China). The samples were subjected to ten-fold serial dilutions in DMEM containing glutamine, $5 \%$ heat-inactivated FBS and $0.05 \%$ gentamicin (Invitrogen Life Technologies). Diluted samples $(0.2 \mathrm{ml})$ were added to each well of cells in duplicate or triplicate. Following sample inoculation, the plates were placed in an incubator (Thermo Scientific 3111; Thermo Fisher Scientific, Waltham, MA, USA) for $1 \mathrm{~h}$ at $37^{\circ} \mathrm{C}$ with $5 \% \mathrm{CO}_{2}$. Every $15 \mathrm{~min}$, the plates were agitated, allowing the sample dilution to move over the monolayer and ensuring full coverage by the inoculum. Following $1 \mathrm{~h}$ of incubation, the wells were covered with $2 \mathrm{ml}$ $1: 1$ primary overlay comprising $1 \%$ agarose (SeaKem ME; $0.5 \%$ final concentration) and $2 \mathrm{X}$ basal medium Eagle with Earle's salts [4-(2-hydroxyethyl)-1-piperazineethanesulfonic acid, $10 \%$ FBS and $0.05 \%$ gentamicin]. The plates were incubated at $37^{\circ} \mathrm{C}$ with $5 \% \mathrm{CO}_{2}$ for seven days. At seven days post-inoculation, $2 \mathrm{ml}$ secondary staining overlay [primary overlay and 5\% Neutral Red stain (Santa Cruz Biotechnology, Inc., Dallas, TX, USA)] was added to each well. At 24 and $48 \mathrm{~h}$, the plaques were counted to determine the sample viral titer in plaque forming units $(\mathrm{Pfu}) / \mathrm{ml}$. The minimum detection limit of the plaque assay was calculated as $<1.666 \mathrm{Pfu}$ based on the observation of one plaque out of three replicates (average $=0.333$ ) counted in a sample of $0.2 \mathrm{ml}$ virus added to cells. The maximum detection limit in the current format was $>5 \times 10^{8} \mathrm{Pfu} / \mathrm{ml}$ [based on the observation of more than 100 plaques in the replicates of the six-fold dilution $\left.\left(100 \times 5 \times 10^{6}\right)\right]$.

Multiplicity of infection. MCF-7 cells were seeded into 24-well plates at $5 \times 10^{4}$ cells/well. pAd-EGFP-T42, pAd-EGFP-4xT42 or pAd-EGFP was added at MOIs of 0, 25, 50, 100 and 200 , respectively (six wells for each MOI). Following $4 \mathrm{~h}$ of incubation, cells were washed twice with $500 \mu \mathrm{l}$ PBS prior to the addition of $500 \mu \mathrm{l}$ DMEM containing $10 \%$ FBS to each well. Following $24 \mathrm{~h}$ of further incubation, the transduction efficiency was evaluated by observing the expression of eGFP.

MTT analysis. Four groups were evaluated in the present study: pAd-EGFP-T42, pAd-EGFP-4xT42, pAd-EGFP control and a blank control group. At $24 \mathrm{~h}$ prior to infection, MCF-7 cells that were in an exponential growth phase were plated in six-well plates at a density of $4 \times 10^{5}$ cells/well. The following day, the cells in each group were transfected and incubated for $18 \mathrm{~h}$. Following incubation, the success of transfection was evaluated by examination of the cells under a fluorescence microscope (Nikon-TE2000-U), and the cells in each group were transferred to 96 -well plates at a density of $2 \times 10^{4}$ cells/well with an MOI of 100 . An MTT assay was subsequently conducted in each group of cells at 12, 24, 36 and
$48 \mathrm{~h}$ following transfection. To perform the MTT assay, $25 \mu \mathrm{l}$ fresh MTT (5 mg/ml; Sigma-Aldrich, St Louis, MO, USA) was added to each well. The cells were then incubated at $37^{\circ} \mathrm{C}$ for $4 \mathrm{~h}$ to allow the incorporation and conversion of MTT to the formazan derivative. The formazan derivative was solubilized by the addition of $100 \mu \mathrm{l}$ DMSO (Sigma-Aldrich). The absorbance values were measured at $570 \mathrm{~nm}$ using an EXL808 plate reader (Bio-Tek Instruments, Inc., Winooski, VT, USA).

Flow cytometric analysis. MCF-7 cells were prepared and separated into four groups according to an identical protocol to that of the MTT assay. Twenty-four hours following confirmation of transfection, $100 \mathrm{MOI}$ recombinant adenovirus or control pAd-EGFP adenovirus was added to each well and the plates were incubated for $4 \mathrm{~h}$ at $37^{\circ} \mathrm{C}$ and $5 \% \mathrm{CO}_{2}$. The cells were then resuspended in ice-cold $70 \%$ ethanol and stored at $-20^{\circ} \mathrm{C}$ prior to analysis. The cellular DNA was stained with PBS containing $100 \mu \mathrm{g} / \mathrm{ml}$ propidium iodide (Beyotime Institute of Biotechnology, Nantong, China), 0.6\% Nonidet P-40 and $100 \mu \mathrm{g} / \mathrm{ml}$ RNase A (Beyotime Institute of Biotechnology). The samples $\left(10^{5}\right)$ were analyzed using a FACS BD Aria flow cytometer at $488 \mathrm{~nm}$ (BD Biosciences, Franklin Lakes, NJ, USA). Ten thousand cells were analyzed using three replicates. The results were analyzed using the FACSDiva ${ }^{\mathrm{TM}}$ version 6.1.1 (BD Biosciences) software.

Western blot analysis. MCF-7 cells were inoculated in six-well plates at $4 \times 10^{5}$ cells/well and treated as aforementioned. A lysis buffer [150 mmol/1 NaCl, $1 \% \mathrm{NP}-40,0.02 \%$ sodium azide, $10 \mu \mathrm{g} / \mathrm{ml}$ phenylmethanesulfonylfluoride and $50 \mathrm{mmol} / \mathrm{l}$ Tris- $\mathrm{HCl}$ ( $\mathrm{pH} 8.0)]$ was added to the cells at $150 \mu \mathrm{l}$ per tube. The lysate was incubated on ice for $30 \mathrm{~min}$ and centrifuged at $12,000 \mathrm{xg}$ for $4 \mathrm{~min}$ at $4^{\circ} \mathrm{C}$. The supernatant was collected and the protein concentration was analyzed using a bicinchoninic acid protein assay kit (Beyotime Institute of Biotechnology). Subsequently, western blot analysis was performed. Briefly, protein samples were electrophoresed by $10 \%$ SDS-PAGE and transferred onto a nitrocellulose membrane (Millipore, Billerica, MA, USA). The membrane was incubated in fresh blocking buffer at room temperature for $1 \mathrm{~h}$ and then probed with the following antibodies [all diluted at 1:1,000, v/v; Beyotime Institute of Biotechnology): Mouse anti-human polyclonal $\beta$-actin,mouse anti-human polyclonalanti-caspase-3, mouse anti-human monoclonal anti-B-cell lymphoma 2 (Bcl-2) and rabbit anti-human polyclonal $\mathrm{Bcl}-2$-associated $\mathrm{X}$ protein in blocking buffer $[0.1 \%(\mathrm{v} / \mathrm{v})$ Tween 20 in Tris-buffered saline, $\mathrm{pH} 7.4$, containing $5 \%(\mathrm{w} / \mathrm{v})$ skimmed milk] at $4^{\circ} \mathrm{C}$ overnight. Following washing three times with Tris-buffered saline containing $0.1 \%$ (v/v) Tween 20 (TBST) buffer for 5 min each, the membrane was incubated with the appropriate horseradish peroxidase-conjugated secondary antibody [goat anti-mouse immunoglobulin G ( $\operatorname{Ig} G), 1: 5,000$; Kangchen Biotechnology, Shanghai, China and goat anti-rabbit IgG, 1:2,000; Santa Cruz Biotechnology, Inc. Dallas, TX, USA] at room temperature for $1 \mathrm{~h}$ and then washed again three times in TBST buffer. The membrane was incubated with enhanced chemiluminescence substrate solution (Santa Cruz Biotechnology, Inc.) for 5 min according to the manufacturer's instructions prior to visualization with autoradiography film (Bio-Rad ChemiDoc XRS; Bio-Rad Laboratories, Hercules, CA, USA). 
Protective effects of pAd-EGFP-T42 and pAd-EGFP-4xT42 on axenograft mouse model. MCF-7 cells in the exponential phase of growth were adjusted to a density of $5 \times 10^{7}$ cells $/ \mathrm{ml}$. Mice were inoculated subcutaneously in the right breast fat pad with $0.1 \mathrm{~m} 1 \mathrm{MCF}-7$ cell suspension and a $10-\mathrm{mm}^{3}$ (volume=long diameter $\mathrm{x}$ short diameter ${ }^{2} \mathrm{x} \pi / 6$ ) tumor formed $\sim$ two weeks subsequently. The nude mice were divided randomly into four groups of ten mice. Then, 1.0x10 $\mathrm{Pfu} / \mathrm{kg}$ pAd-EGFP-T42, pAd-EGFP-4xT42 or pAd-EGFP was injected locally into the tumor on days one, five and ten. For the control group, PBS $(0.1 \mathrm{ml}$ total $)$ was injected locally into the tumor on an identical treatment schedule. The tumor was dissected for measurement 28 days after the first injection and the growth inhibition ratio of the tumor was calculated using the following equation: Growth inhibition ratio of the tumor=[1-(size of tumor of each group on day 28 -size on day 0 )/(size of tumor of the blank control group on day 28 -size on day 0)] x100\%.

Histological examination. The entire tumor was embedded in paraffin. Paraffin sections were subsequently dewaxed and antigen retrieval was performed by incubating the sections in $0.01 \mathrm{~mol} / \mathrm{l}$ citrate buffer $(\mathrm{pH} 6.0)$ at $92-98^{\circ} \mathrm{C}$. The sections were then incubated in $0.05 \%$ trypsin (Beyotime Institute of Biotechnology) at $37^{\circ} \mathrm{C}$ for $30 \mathrm{~min}$ followed by incubation in $3 \% \mathrm{H}_{2} \mathrm{O}_{2}$ (Beyotime Institute of Biotechnology) for $10 \mathrm{~min}$. Primary labeling was conducted using mouse anti-human CD34 monoclonal antibody (Sigma-Aldrich) at a dilution of 1:100 at $4^{\circ} \mathrm{C}$ overnight. Following rewarming of the sections, the secondary antibody was added to the slides followed by 3,3'-diaminobenzidine for a color reaction. The sections were subsequently stained with hematoxylin. PBS was used in place of the primary antibody as a negative control. To quantify the vascular area, three areas of high vascular density were selected at low magnification (x40) and the microvessels were quantified in each region at high magnification (x200). The average for each section was considered to indicate the microvascular density.

Statistical analysis. Values are presented as the mean of $\geq$ three independent experiments \pm standard deviation unless otherwise stated. An unpaired Student's t-test or analysis of variance was used to determine the significance of differences between experimental groups. The statistical analyses were performed using SPSS version 13.0 software (SPSS Inc., Chicago, IL, USA). P $<0.05$ was considered to indicate a statistically significant difference between values.

\section{Results}

Successful construction of pAd-EGFP-T42 and $p A d-E G F P-4 x T 42$. The pAd-EGFP-T42 and pAd-EGFP-4xT42 plasmid DNA was extracted and digested using Pac I. Following Pac I digestion, the fragment sizes were determined as $3,000 \mathrm{bp}$ and $>15 \mathrm{~kb}$, which confirmed that the plasmid backbone was pAd-BL-dest and that the pAd-EGFP-T42 and pAd-EGFP-4xT42 inserts were correct (20).

Transfection of 293 cells with pAd-EGFP-4xT42. Following $24 \mathrm{~h}$ of transfection with pAd (empty vector), pAd-EGFP-T42 or pAd-EGFP-4xT42, the virus demonstrated a cytopathic effect on the transfected 293 cells and EGFP was successfully identified by fluorescent microscopy (Fig. 1). Consistent with these results, RT-PCR of the pAd-EGFP-T42 and pAd-EGFP-4xT42 adenoviruses indicated that T42/4xT42 was expressed following transfection. The virus titer of the 293 cells was also measured at $5 \times 10^{9} \mathrm{Pfu} / \mathrm{ml}$.

pAd-EGFP-4xT42 and pAd-EGFP-T42 enhance tumor cell growth inhibition rate. The cell grow th inhibition rate was significantly enhanced in the pAd-EGFP-T42 and pAd-EGFP-4xT42 transfection groups compared with that of the control group at 36 and $48 \mathrm{~h}(\mathrm{P}<0.05)$. However, there was no significant difference in the cell growth inhibition rate between the pAd-EGFP plasmid transfection group and that of the control group at any time-point (Fig. 2).

pAd-EGFP-4xT42 and pAd-EGFP-T42 enhance MCF-7 cell apoptosis. The results of the flow cytometric analysis of apoptosis are exhibited in Fig. 3. The apoptotic rates of the blank control, pAd-EGFP, pAd-EGFP-T42 and pAd-EGFP-4xT42 groups were $11.82 \pm 2.31,14.86 \pm 1.02,39.38 \pm 2.17$ and $42.29 \pm 3.26 \%$, respectively. The apoptotic rates of the MCF-7 cells infected with pAd-EGFP-T42 or pAd-EGFP-4xT42 were significantly higher than those of the cells infected with the control adenovirus and the non-transfected cells in the blank control group $(\mathrm{P}<0.05)$. There was no significant difference in the apoptotic rate of the control group and that of the pAd-EGFP group $(\mathrm{P}>0.05)$ or between that of the pAd-EGFP-T42 group and that of the pAd-EGFP-4xT42 group.

pAd-EGFP-T42 and pAd-EGFP-4xT42 increases pro-apoptotic protein expression in MCF-7 cells. Western blot analysis was used to evaluate the expression levels of apoptosis-associated proteins in MCF-7 cells treated with pAd-EGFP-T42 and pAd-EGFP-4xT42. As indicated in Fig. 4, expression levels of Bcl-2 were significantly decreased, while expression levels of Bax were significantly enhanced in MCF-7 cells treated with pAd-EGFP-T42 and pAd-EGFP-4xT42 in comparison to those of the control and pAd-EGFP groups. The levels of the active $17 \mathrm{kDa}$ cleaved caspase-3 fragment were significantly increased in MCF-7 cells transfected with pAd-EGFP-T42 and pAd-EGFP-4xT42, compared with those of the control and pAd-EGFP groups.

Protective effects of pAd-EGFP-T42 and pAd-EGFP-4xT42 on a xenograft mouse model. Mice were sacrificed by cervical dislocation 28 days after injection of adenovirus, and the tumors were excised for measurement. The average tumor sizes in each of the four groups were $122.46 \pm 8.08$, $113.28 \pm 6.37,63.32 \pm 13.10$ and $54.86 \pm 13.59 \mathrm{~mm}^{3}$, respectively. The inhibition rates of tumor growth are presented in Fig. 5 . There was a significant increase in the tumor growth inhibition rate of the pAd-EGFP-T42- and pAd-EGFP-4xT42-transfected groups and that of the control group $(\mathrm{P}<0.05)$. There was also a significant increase in the tumor growth inhibition rate of the pAd-EGFP-4xT42 group compared to that of the pAd-EGFP group $(\mathrm{P}<0.05)$.

pAd-EGFP-T42 and pAd-EGFP-4xT42 decrease tumor microvessel density. Irregular neovascularization was detected 
A
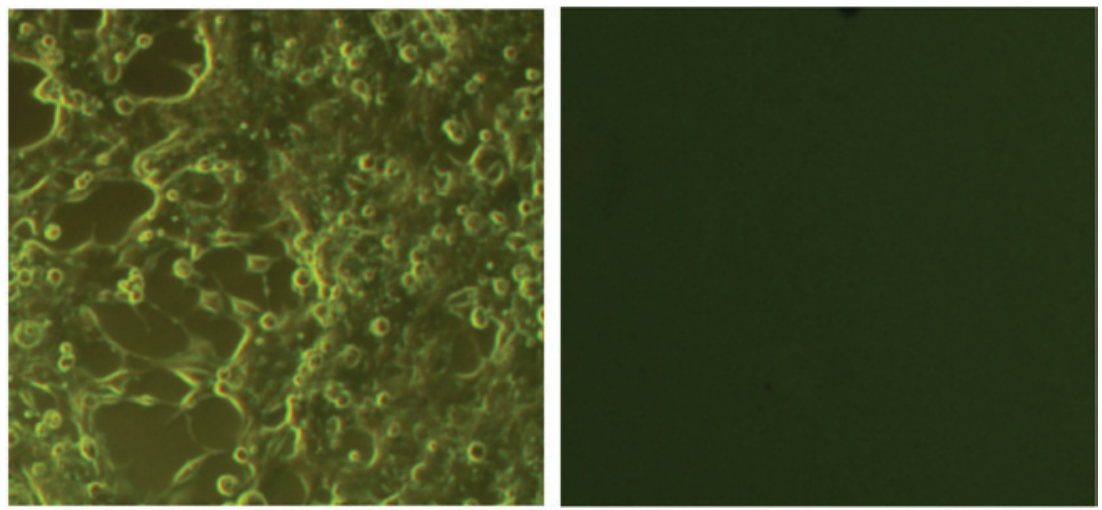

$\mathbf{B}$
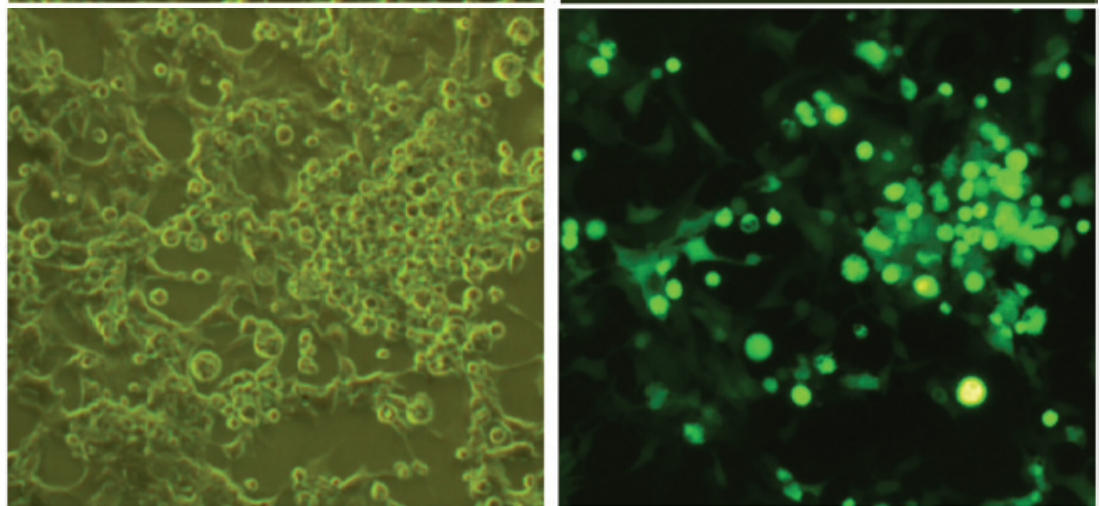

C
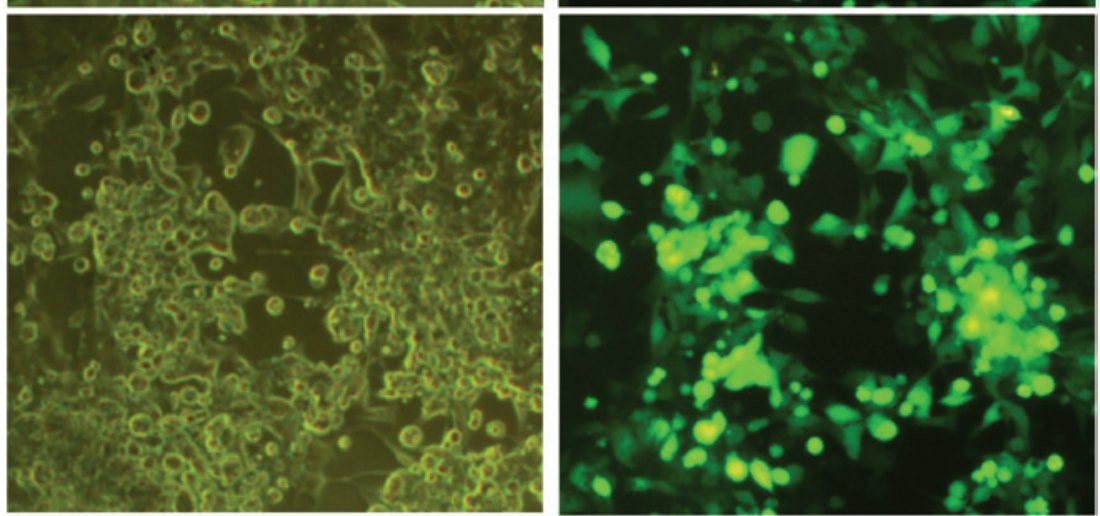

Figure 1. Expression of GFP following pAd, pAd-EGFP-T42 or pAd-EGFP-4xT42 transfection of 293 cells. (A) GFP expression following pAd transfection. (B) GFP expression following pAd-EGFP-T42 transfection. (C) GFP expression following pAd-EGFP-4xT42 transfection. Magnification, x100. White light field on the left, green fluorescence field on the right. EGFP, enhanced green fluorescent protein; pAd, plasmid adenovirus.

in the tumor tissue, in particular at the edge of the tumor, by immunohistochemical staining. In the tumors of the mice infected with pAd-EGFP-T42 and pAd-EGFP-4xT42, there was a decreased microvessel density compared to that of the tumors of the mice transfected with pAd-EGFP. The results of the microvessel count are presented in Table I and Fig. 6.

\section{Discussion}

In 2000, Maeshima et al (10) reported that tumstatin was able to selectively inhibit the angiogenesis of tumor tissue, without influencing normal angiogenesis. Tumstatin has been described as an endogenous antitumor agent $(11,21)$. Amino acids 54-132 at the N-terminus of the protein have demonstrated anti-angiogenic effects, while amino acids 185-203 at the C-terminus have displayed antitumor activity (21).

Through binding with $\alpha \mathrm{V} \beta 3$ integrin, tumstatin inhibits activation of the transduction pathway involving focal adhesion

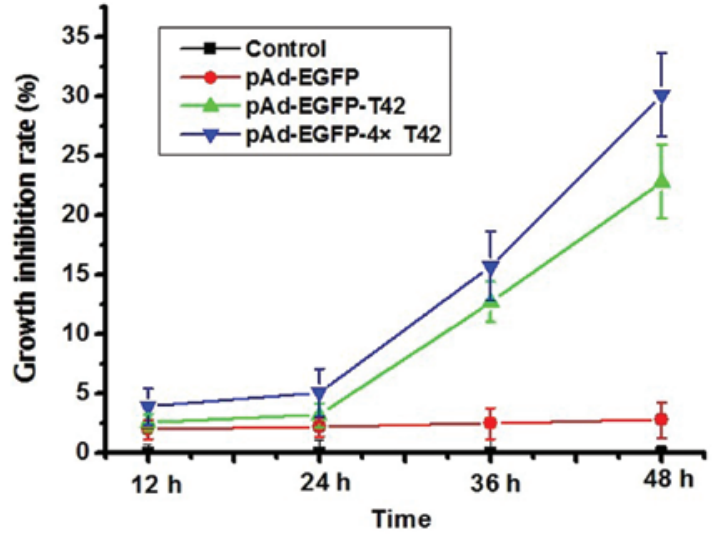

Figure 2. Comparison of growth inhibition rate between the transfected and control groups. Changes in cell survival determined by MTT assay following incubation for various times with untreated (control), pAd-EGFP, pAd-EGFP-T42 or pAd-EGFP-4xT42-treated cells (control=100\% survival). The results are presented as the mean of three independent experiments. EGFP, enhanced green fluorescent protein; pAd, plasmid adenovirus. 
A

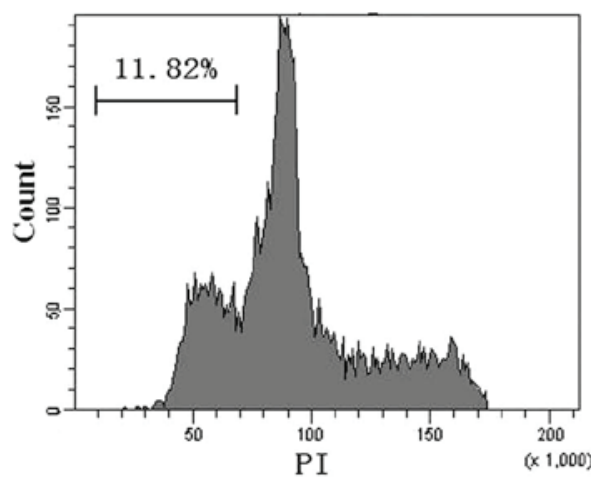

C

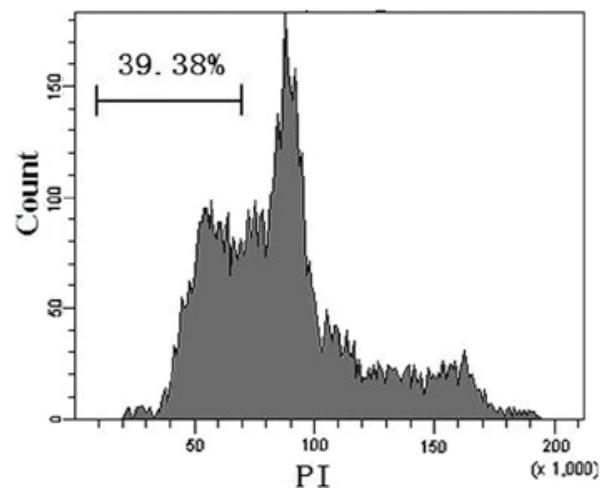

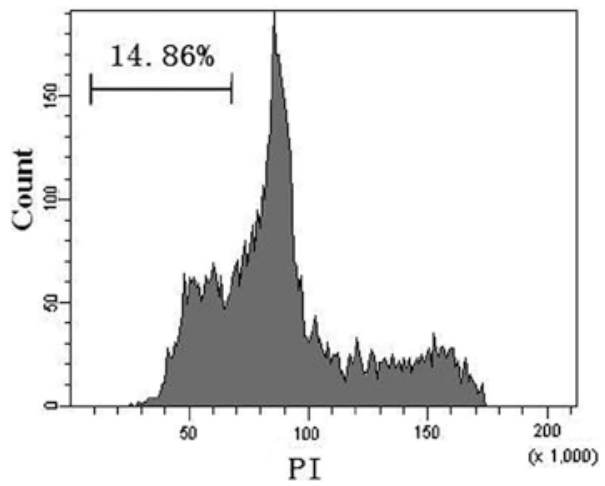

D

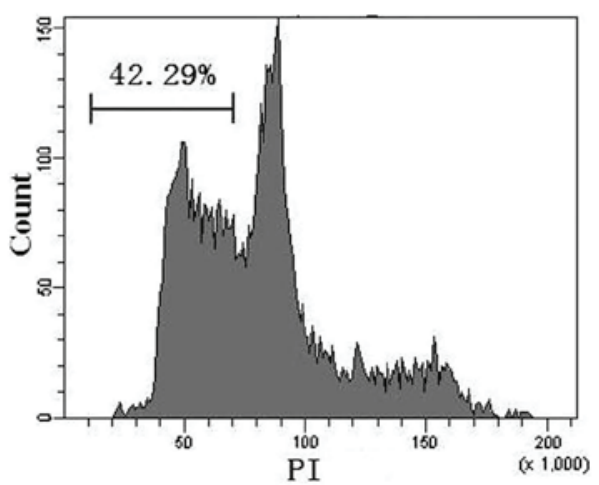

Figure 3. Flow cytometric analysis of untreated, pAd-EGFP-, pAd-EGFP-T42- and pAd-EGFP-4xT42-induced apoptosis in MCF-7 cells. DNA was stained with propidium iodide. Rate of apoptosis was indicated by the sub-G1 population. (A) Control (untreated) group. (B) pAd-EGFP infection group. (C) pAd-EGFP-T42 infection group. (D) pAd-EGFP-4xT42 infection group. EGFP, enhanced green fluorescent protein; pAd, plasmid adenovirus.

A

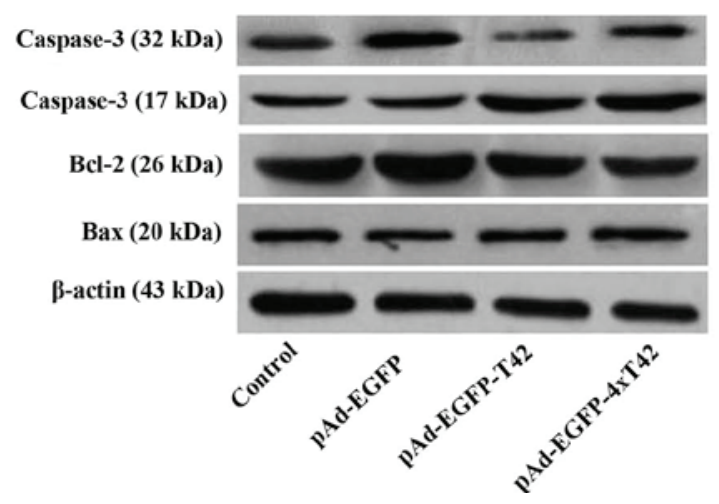

B

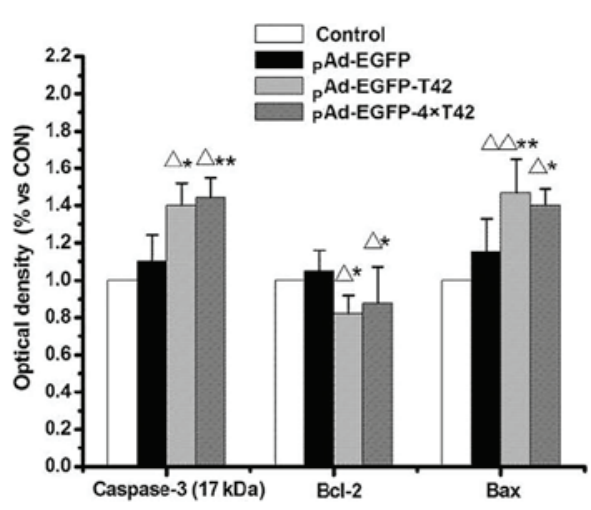

Figure 4. pAd-EGFP-T42 and pAd-EGFP-4xT42 increase anti-apoptotic protein expression and decrease pro-apoptotic protein expression. (A) Representative western blot indicating Bcl-2, Bax and caspase-3 cleavage expressions in MCF-7 cells that were incubated with pAd-EGFP,pAd-EGFP-T42 or pAd-EGFP-4xT42. (B) Bar charts indicating the different intensities of apoptotic proteins between four groups. Results were normalized against $\beta$-actin and expressed as the fold change compared to that of the control (untreated) group, respectively. Values are expressed as the mean \pm standard error of the mean $(\mathrm{n}=3) .{ }^{\Delta} \mathrm{P}<0.05,{ }^{\Delta \Delta} \mathrm{P}<0.01$ vs. control group; "P<0.05, ${ }^{* *} \mathrm{P}<0.01$ vs. pAd-EGFP group. EGFP, enhanced green fluorescent protein; pAd, plasmid adenovirus; Bcl-2, B-cell lymphoma 2; Bax, Bcl-2-associated X protein.

kinase, phosphatidylinositol-4,5-bisphosphate 3-kinase and Akt and the mammalian target of rapamycin pathway (22). As a result, tumstatin prevents the dissociation of eukaryotic initiation factor $4 \mathrm{E}$ protein from $4 \mathrm{E}$-binding protein 1 , which leads to the inhibition of cap-dependent protein synthesis in endothelial cells (23). Twenty-one peptides function to preserve the anti-angiogenic activity of tumstatin (24). Studies have also demonstrated that 19 peptides are involved in the inhibition of adhesion, chemotaxis and proliferation of various cells by binding to the CD47/integrin-associated protein and $\alpha v \beta 3$ proteins on the surface of tumor cells and forming a complex (25). In the present study, the 19-residue tumstatin peptide, a fragment with antitumor activity, and the 21-residue peptide were ligated. The RGD structure was also added, as well as amino acids -GG-, to form a 21 peptide-GG-19 peptide construct, which consisted of 42 peptides in total. RGD has been shown to inhibit the proliferation and migration of tumor cells and was therefore predicted to increase the antitumor capability of the 42-residue peptide (26). These small peptides from tumstatin promoted cell apoptosis via extracellular effects. However, to the best of our knowledge, it had not previously been elucidated whether the tumstatin T42 peptide 
Table I. Neo-angiogenesis density in tumors of various groups.

\begin{tabular}{lcccc}
\hline Group & Blank control & pAd-EGFP & pAd-EGFP-T42 & pAd-EGFP-4xT42 \\
\hline Microvessel density & - & $18.88 \pm 2.53$ & $12.71 \pm 3.48$ & $11.97 \pm 3.31$ \\
\hline
\end{tabular}

EGFP, enhanced green fluorescent protein, pAd, plasmid adenovirus. Values are presented as the mean \pm standard deviation of three independent experiments.

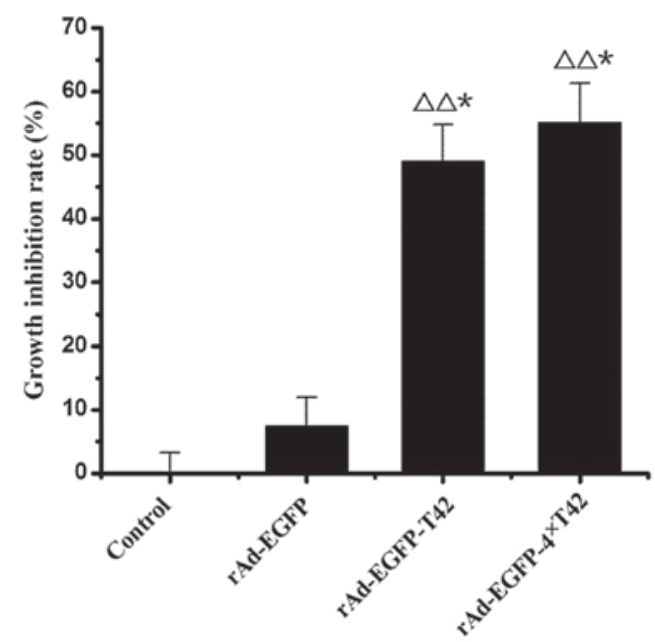

Figure 5. Tumor growth inhibition rate in vivo. The growth inhibition rates of the pAd-EGFP-T42 and pAd-EGFP-4xT42 groups were significantly higher than those of the control (phosphate-buffered saline-treated) group. Significant differences were also observed between the pAd-EGFP-T42 and pAd-EGFP-4xT42 groups and that of the pAd-EGFP group $(\mathrm{P}<0.05)$; There was no difference between the growth inhibition rate of the control group and that of the pAd-EGFP group $(\mathrm{P}>0.05)$ or between the pAd-EGFP-T42 and pAd-EGFP-4xT42 groups. EGFP, enhanced green fluorescent protein; pAd, plasmid adenovirus. Values are presented as the mean \pm standard deviation of three independent experiments. ${ }^{*} \mathrm{P}<0.05$ vs. pAd-EGFP group; ${ }^{\Delta \Delta} \mathrm{P}<0.01$ vs. control group.

was able to influence the intracellular activity of tumor cells. Therefore, in the present study, eukaryotic cells were infected with an adenovirus carrying the target gene and the expression levels of target proteins in these eukaryotic cells were subsequently evaluated. In addition, the antitumor effects of the T42 peptide were further examined by infecting breast cancer cells with an adenovirus carrying the T42 target gene.

The adenovirus Ad5, selected for use in the present study, has been improved by advancements in biotechnology. The Ad5 genome cannot produce the packaging protein E1 unless it is provided by the host 293 cells; therefore, its genome is able to be amplified in 293 cells (27). However, normal human tissue cells do not express the E1 protein; therefore, the packaging protein E1 of Ad5 will not be present and the virus will be unable to proliferate in human cells (28). The virus is capable of being engineered to produce a specific target protein, so it is safe for use in gene therapy research (29). Following the construction of the adenoviral vector containing the T42 or 4xT42 gene coupled with EGFP, GFP was observed using fluorescence microscopy, which confirmed that the EGFP fragment had been successfully incorporated into the 293 cells. RT-PCR verified that the T42 and 4xT42 plasmid were expressed by 293 cells. Furthermore,
A

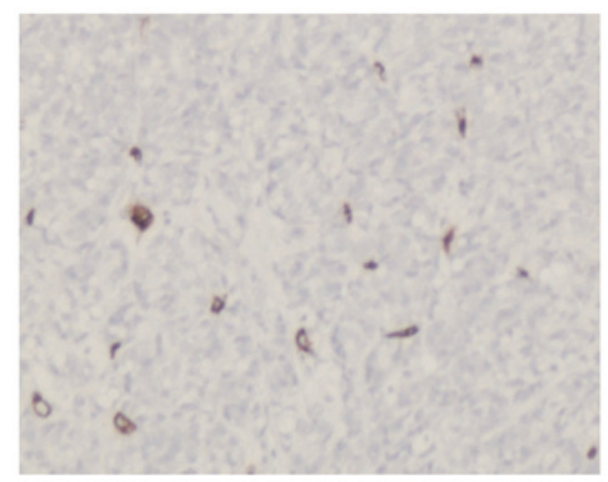

B

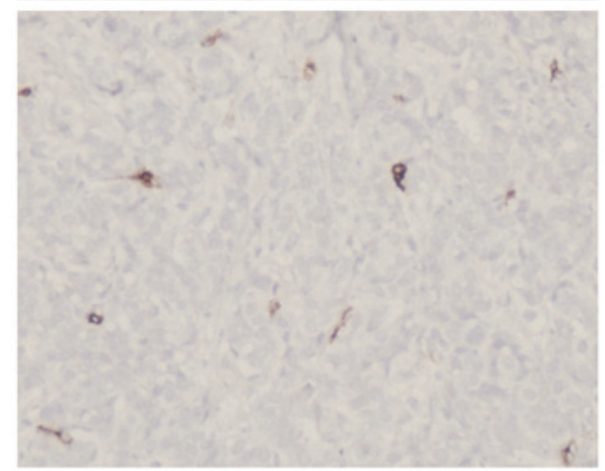

C

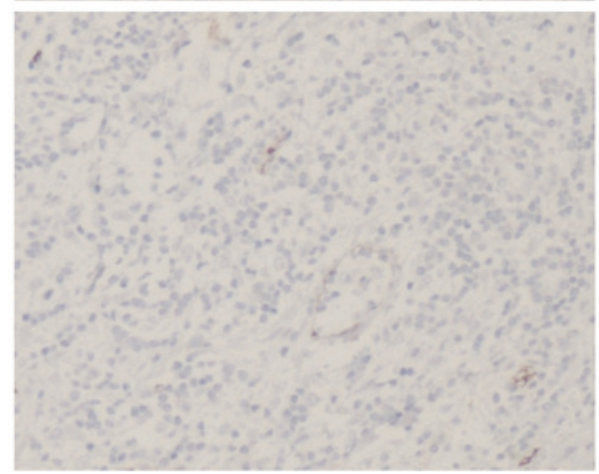

D

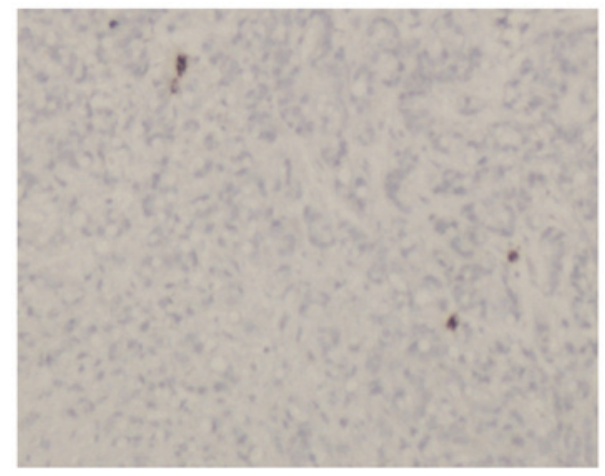

Figure 6. Anti-CD34 staining of mouse tumors following various treatments. (A) Control (phosphate-buffered saline-treated). (B) pAd-EGFP infection group. (C) pAd-EGFP-T42. (D) pAd-EGFP-4xT42 infection group. Magnification, x40. EGFP, enhanced green fluorescent protein; pAd, plasmid adenovirus. 
the fluorescence observed following transfection indicated that the adenoviruses were successfully packaged and transfected effectively. At seven days post-transfection, a large proportion of the 293 cells shed and appeared disintegrated under microscopic examination, which was consistent with previous studies (30). When transfections are performed without the use of a fluorescent indicator, the amount of time in which viruses are produced is difficult to accurately determine. By contrast, in the present study, it was ensured that the maximum viral titer and conditions for viral amplification were optimized.

The successful construction and packaging of the pAd-EGFP-T42 and pAd-EGFP-4xT42 adenoviruses and the preliminary results regarding their function indicated that the adenovirus promoted apoptosis and inhibited the growth of cancer cells, which provided a foundation for further investigation into gene therapies for cancer. The growth inhibition ratios of the transfected groups compared to those of the control group at four different time-points were calculated using the MTT assay. The results of these experiments demonstrated that no significant inhibitory effect of the pAd-EGFP-T42 and pAd-EGFP-4xT42s plasmid on the growth inhibition rate was evident at 12 or $24 \mathrm{~h}$ in comparison to that of the control group. However, the inhibitory effect increased over time, and at 36 and $48 \mathrm{~h}$ there was a significant difference between the growth inhibition rate of pAd-EGFP-T42 and pAd-EGFP-4xT42 plasmid groups and that of the control group $(\mathrm{P}<0.05)$, while there was no significant difference between that of the pAd-EGFP plasmid group and that of the control group.

Flow cytometry was used to determine the apoptotic rates of the cells in the recombinant adenovirus pAd-EGFP-T42 and pAd-EGFP-4xT42 groups, the pAd-EGFP adenovirus control group and the blank control group. The apoptotic rates of the cells infected with pAd-EGFP-T42 and pAd-EGFP-4xT42 were significantly higher than those of the cells infected with the control adenovirus or the non-transfected cells in the blank control group $(\mathrm{P}<0.05)$. These results demonstrated that transfection of pAd-EGFP-T42 and pAd-EGFP-4xT42 were able to significantly enhance the apoptotic rate of tumor cells at $48 \mathrm{~h}$ post-transfection, which was consistent with the results of the MTT assay.

The results of the present study revealed that pAd-EGFP-T42 and pAd-EGFP-4xT42 suppressed the growth of MCF-7 cells in vitro and the growth of tumor xenografts in vivo. In MCF-7 cells treated with pAd-EGFP-T42 or pAd-EGFP-4xT42, there was significant downregulation of Bcl-2 expression and upregulation of Bax and caspase- 3 expression, which are key executors during apoptosis. These results indicated that the mitochondrial pathway may be involved in regulating the induction of apoptosis (31). There are distinct mechanisms that mediate the anti-angiogenic and antiproliferative activities of these tumstatin peptides. Mice treated with pAd-EGFP-T42 or pAd-EGFP-4xT42 had fewer CD34-positive vessels. The results observed were similar to those of previous reports indicating that the 19 peptide fragments were able to inhibit tumor proliferation and the 21 peptide fragments were able to inhibit endothelial cell proliferation. Therefore, the recombinant adenovirus packaged in the present study had both abilities.

Recombinant adenoviruses carrying the T42 or $4 \mathrm{xT} 42$ genes (pAd-EGFP-T42/pAd-EGFP-4xT42) were successfully constructed. The results of the present study revealed that the adenoviruses constructed were able to inhibit the proliferation of breast cancer cells in vivo and in vitro. Of note, no significant difference was observed between the pAd-EGFP-T42- and the pAd-EGFP-4xT42-transfected groups. pAd-EGFP-T42 and pAd-EGFP-4xT42 may function via the mitochondrial pathway to inhibit transplant tumor angiogenesis. The adenoviral constructs exhibited antitumor effects when the peptide was expressed in the cells in vitro, and also upon the expression of the peptide gene by the recombinant virus in vivo. However, the target of the T42 peptide remains to be elucidated. In conclusion, the results of the present study indicated the relevance of the T42 peptide in the development of novel cancer treatment strategies, which requires further study in order to elucidate the mechanism of the antitumor effects of this adenovirus.

\section{Acknowledgements}

The authors would like to thank the Science and Technology Funds of the Hei Longjiang Education Department of China for the grant they provided to support the present study (no. 12511188).

\section{References}

1. Tazawa H, Kagawa $\mathrm{S}$ and Fujiwara T: Advances in adenovirus-mediated p53 cancer gene therapy. Expert Opin Biol Ther 13: 1569-1583, 2013.

2. Liu SX, Xia ZS and Zhong YQ: Gene therapy in pancreatic cancer. World J Gastroenterol 20: 13343-13368, 2014

3. Folkman J: Tumour angiogenesis: therapeutic implications. N Engl J Med 285: 1182-1186, 1971.

4. Hlatky L, Hahnfeldt P and Folkman J: Clinical application of antiangiogenic therapy: microvessel density, what it does and doesn't tell us. J Natl Cancer Inst 94: 883- 893, 2002.

5. Albig AR and Schiemann WP: Fibulin-5 antagonizes vascular endothelial growth factor (VEGF) signaling and angiogenic sprouting by endothelial cells. DNA Cell Biol 23: 367-379, 2004.

6. Sim BK: Angiostatin and endostatin: endothelial cell-specific endogenous inhibitors of angiogenesis and tumor growth. Angiogenesis 2: 37-48, 1998.

7. Ribatti D: History of research on angiogenesis. Chem Immunol Allergy 99: 1-14, 2014.

8. Hamano Y, Zeisberg M, Sugimoto H, et al: Physiological levels of tumstatin, a fragment of collagen IV alpha3 chain, are generated by MMP-9 proteolysis and suppress angiogenesis via alphaVbeta3 integrin. Cancer Cell 3: 589-601, 2003.

9. Su XJ, Wu FJ, Yuan LJ and Lin XS: Determination of anti-Hepg2 cells activity and purification of tumor chalone T42 peptide. J Jilin Univ, Med Ed 36: 86-89, 2010.

10. Maeshima Y, Colorado PC, Torre A, et al: Distinct antitumor properties of a type IV collagen domain derived from basement membrane. J Biol Chem 275: 21340-21348, 2000.

11. Maeshima Y, Manfredi M, Reimer C, et al: Identification of the anti-angiogenic site within vascular basement membrane-derived tumstatin. J Biol Chem 276: 15240-15248, 2001.

12. Saus J, Wieslander J, Langeveld JP, Quinones S and Hudson BG: Identification of the Goodpasture antigen as the alpha 3(IV) chain of collagen IV. J Biol Chem 263: 13374-13380,1988.

13. Wang SJ, Liu XH, Ji YB and Chen N: Study on Biological Activity of Two Modified Anti-tumor Peptide of Tumstatin. Biochemistry and Biophysics 34: 1152-1161, 2007 (In Chinese).

14. Gao Y, Yu Y, Lu S, Sun BC, Wang XH: Cloning and expression of human tumstatin gene in E. Coli. Chinese Journal of Biochemical Pharmaceutics 26: 324-327, 2005 (In Chinese).

15. Li Y, Liu XH, Lin XS, et al: Inhibitory effect of tumstatin related peptide T42 on human umbilical vein endothelial cells and human gastric adenocarcinoma. J Clin Rehab Tissue Engineer Res 11: 1837-1840, 2007 (In Chinese).

16. Su XJ, Lin XS, Wu FJ and Yuan LJ: Antitumor activity of tumor chalone 42 peptide combined with cyclophosphamide. China Cancer 5: 334-337, 2010. 
17. Jones MS II, Harrach B, Ganac RD, et al: New adenovirus species found in a patient presenting with gastroenteritis. J Virol 81: 5978-5984, 2007.

18. Bernt KM, Ni S, Tieu AT and Lieber A: Assessment of a combined, adenovirus-mediated oncolytic and immunostimulatory tumor therapy. Cancer Res 65: 4343-4352, 2005.

19. Takehashi M, Kanatsu-Shinohara M, Inoue $K$, et al Adenovirus-mediated gene delivery into mouse spermatogonial stem cells. Proc Natl Acad Sci USA 104: 2596-2601, 2007.

20. Qi DD, Chen YL, Guo JZ, Zhu GM, Zhang T, Jiang S and Wang YZ: Constructiong of recombinant adenovirus vector with $4 \mathrm{~T} 42$ peptide gene, and study of the effect of anti- SKBR3 cell in vitro. Chinese Journal of Gerontology 32: 2781-2783, 2012 (In Chinese)

21. Maeshima Y, Yerramalla UL, Dhanabal M, et al: Extracellular matrix-derived peptide binds to alpha(v)beta(3) integrin and inhibits angiogenesis. J Biol Chem 276: 31959-31968, 2001.

22. Kawaguchi T, Yamashita Y, Kanamori M, et al: The PTEN/Akt pathway dictates the direct alphaVbeta3-dependent growth-inhibitory action of an active fragment of tumstatin in glioma cells in vitro and in vivo. Cancer Res 66: 11331-11340, 2006

23. Maeshima Y, Sudhakar A, Lively JC, et al: Tumstatin, an endothelial cell-specific inhibitor of protein synthesis. Science 295: 140-143, 2002.

24. Zhang GM, Zhang YM, Fu SB, et al: Effects of cloned tumstatin-related and angiogenesis-inhibitory peptides on proliferation and apoptosis of endothelial cells. Chin Med J (Engl) 121: 2324-2330, 2008.
25. Shahan TA, Ziaie Z, Pasco S, et al: Identification of CD47/integrin-associated protein and alpha(v)beta3 as two receptors for the alpha3(IV) chain of type IV collagen on tumor cells. Cancer Res 59: 4584-4590, 1999.

26. Moreno-Manzano V, Lucio-Cazana J, Konta T, Nakayama K and Kitamura M: Enhancement of TNF-alpha-induced apoptosis by immobilized arginine-glycine-aspartate: involvement of a tyrosine kinase-dependent, MAP kinase-independent mechanism. Biochem Biophys Res Commun 277: 293-298, 2000.

27. Spector DJ, Halbert DN and Raskas HJ: Regulation of integrated adenovirus sequences during adenovirus infection of transformed cells. J Virol 36: 860-871,1980.

28. Graham F L, Smiley J, Russell W C, and Nairn R: Characteristics of a human cell line transformed by DNA from human adenovirus type 5. J Gen Virol 36: 59-74, 1977.

29. Silman NJ and Fooks AR: Biophysical targeting of adenovirus vectors for gene therapy. Curr Opin Mol Ther 2: 524-531, 2000.

30. He TC, Zhou S, da Costa LT, Yu J, Kinzler KW and Vogelstein B A simplified system for generating recombinant adenoviruses. Pro Natl Acad Sci USA 95: 2509-2514, 1998.

31. Li YJ, Sun LC, He Y, et al: The anti-tumor properties of two tumstatin peptide fragments in human gastric carcinoma. Acta Pharmacol Sin 30: 1307-1315, 2009. 